\title{
Architecting Communication Network of Networks for Space System of Systems
}

\author{
Kul B. Bhasin \\ NASA Glenn Research Center \\ Cleveland, $\mathrm{OH}$, USA \\ Kul.B.Bhasin@nasa.gov
}

\author{
Jeffrey L. Hayden \\ Principal \\ PresciPoint Solutions, L.L.C. \\ Littleton, CO, USA \\ jlhayden@earthlink.net
}

\begin{abstract}
The National Aeronautics and Space Administration (NASA) and the Department of Defense (DoD) are planning Space System of Systems (SoS) to address the new challenges of space exploration, defense, communications, navigation, Earth observation, and science. In addition, these complex systems must provide interoperability, enhanced reliability, common interfaces, dynamic operations, and autonomy in system management. Both NASA and the DoD have chosen to meet the new demands with high data rate communication systems and space Internet technologies that bring Internet Protocols $(I P)$, routers, servers, software, and interfaces to space networks to enable as much autonomous operation of those networks as possible. These technologies reduce the cost of operations and, with higher bandwidths, support the expected voice, video, and data needed to coordinate activities at each stage of an exploration mission. In this paper, we discuss, in a generic fashion, how the architectural approaches and processes are being developed and used for defining a hypothetical communication and navigation networks infrastructure to support lunar exploration. Examples are given of the products generated by the architecture development process.
\end{abstract}

Keywords: Architecting, space communications, system of systems, SoS, network of networks, NoN, Internet Protocol, IP, DoDAF, architecture framework.

\section{Introduction}

Communications and navigation networks are architected, designed, developed, and deployed based on the type of Space System of Systems (SoS) services needed. Communications and navigation networks of the future are being architected as an integrated set of new assets and a federation of upgraded legacy systems capable of routing IP traffic from any node to any other node on the network [1]. The utilization of upgraded legacy systems as part of the Network of Networks reduces both risk and cost. The Network of Networks will provide the intercommunication support for the Space SoS with each system utilizing a Local Access Network (LAN) for intracommunication support. IP provides a common routable end-to end capability. The use of registries and data dictionaries will provide a structure for the application layer system service interfaces and information exchanges.
This paper focuses on the Network of Networks and space communication links in support of the Space SOS services. NASA and the DoD have chosen IP coupled with modern high bandwidth microwave and optical communication links as the preferred technologies for providing the capabilities, flexibilities, autonomy, and endto-end connectivity needed for space exploration and for supporting the warfighter in theater. There are certain to be significant advances in communications and navigation during the period of time for implementing the networks being defined by the architecture work. However, it is assumed, for this paper, that the advances in technology will be centered on improving information bandwidth with higher frequencies of transmission and improved techniques for modulation and autonomous network detection/connection and on improving IP performance and capability. Thus in the interest of describing a workable architecture, this paper does not attempt to assume technologies that might render IP obsolete. As it is, the networking packet protocol technologies (of nearly 40 years ago) that became IP are advancing and becoming ubiquitous throughout all modes of communication. In addition, while the protocols become more complex as their capabilities are improved, processing and bandwidth also improve, resulting in higher overall network capability. It is not likely that IP will be displaced as a base technology in the timeframe for implementing the new networks.

Figure 1 depicts a complex yet generic set of SoS node types that are expected in missions of exploration. The bottom of the figure indicates the Earth ground systems involved in support of exploration missions. Legacy systems shown include the Deep Space Network (DSN) and its control center, the Tracking and Data Relay Satellite System (TDRSS) Space Network (SN) and its controlling ground stations, and the navigation support center. The communication user control centers are shown for exploration missions, launch, and the International Space Station (ISS). A new distant IP network (DIN) control center (DINCC) and its ground stations (DGS) around the Earth are included in the diagram. The DGSs may be collocated at the DSN sites; however, they will likely require new system hardware and antennas to handle high communication data rates at long distances, new navigational services, and modern networking technologies. This new or highly modified legacy equipment must be dedicated full time to the exploration missions. These DGSs will handle direct communications 


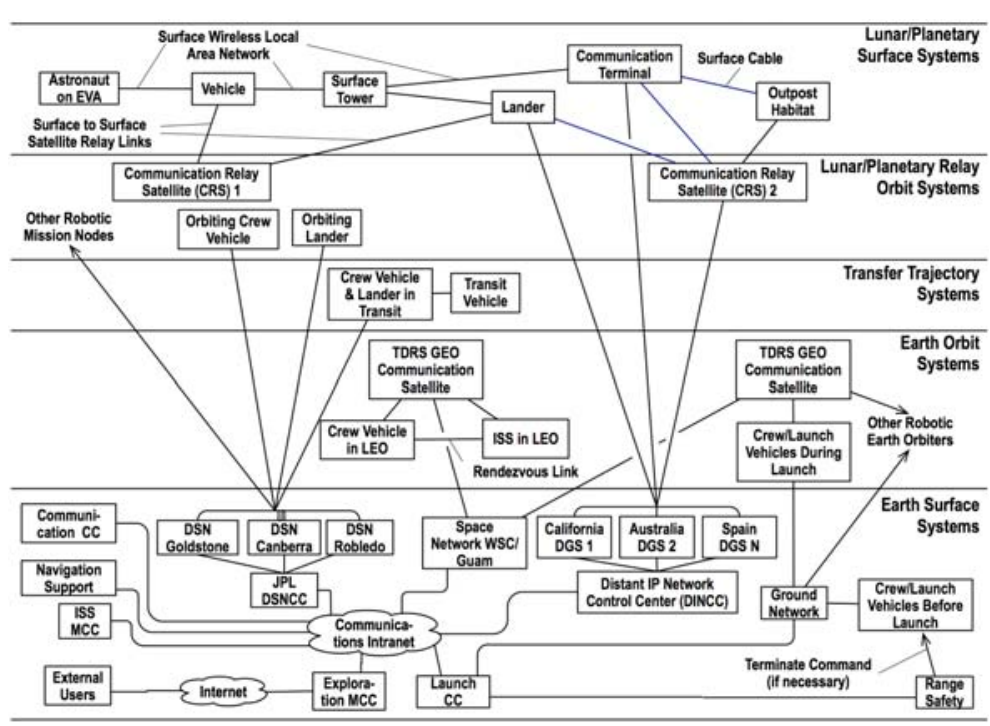

Figure 1. General diagram of communication and navigation systems for future exploration.

and control of communication relay satellites in orbit around the solar system body, as well as direct communications to any visible lunar or planetary surface communication terminal.

As shown at the lower right in the figure, ground network sites handle capturing data from the crew launch vehicle before and during launch. Range Safety is also onhand to send a flight termination signal in the event the launch goes astray. Additionally, a contingency voice system is available in the event normal communication is lost to the flight vehicle.

The next level up, the Earth Orbit Systems, displays the communications needed at that level. Communication support needs are provided by the legacy TDRSS to support crew vehicle rendezvous with the ISS as the crew vehicle replaces the Shuttle in replenishing and recrewing the ISS. The SN can also support the launch vehicle and crew vehicle during launch and rendezvous of the crew vehicle with the lander and Earth departure rocket to get ready for the transfer to the Moon, Mars, or elsewhere. Finally, the SN can support communications to the Crew return capsule during descent and landing.

The third level (Transfer Trajectory Systems) shows a crew vehicle and lander in transit to the solar system object. The legacy DSN system can be modified to provide communications to support this phase and also the in-orbit crew vehicle and lander, shown before landing on the solar system object at level 4 (Lunar/Planetary Relay Orbit Systems).

The fourth level also includes communication relay satellites (CRS) in orbit around the Moon or Mars. The CRSs support communication networking among surface nodes that are spread widely apart, such as astronauts, surface vehicles, and fixed habitats. They also provide high-rate trunks to route communications to and from Earth. The CRSs may include crosslinks among them to route networks from the backside to the front side of the body being explored [2].
The fifth level shows communications with the relay satellites, the Earth's surface, and over the surface of the solar system body. The network over the surface will be of short range and limited by line of sight. However, the surface local area networks can be extended with towers similar to the architecture used in cell phone networks on Earth [3].

It is imperative to implement a SoS at the lowest cost of operations in an infrastructure for space exploration. Automation of ground systems, modernization of legacy assets, highly capable new assets that can be upgraded on the ground, in space, and on the surface of the Moon with software uploads, autonomy of operations of inspace communications assets, and fully IPcompliant networking technologies will be necessary in lowering the cost of operations [4]. Cost of development will not be insignificant, but in the very extended long-range plans being considered, the cost of operations will dwarf the cost of improvement in technologies. Much of the cost of the new technologies has already been carried by the commercial world. IP networking solutions are largely available for direct use. Work in this area does need to be done to handle IP addressing and networking among moving nodes and the distance time delays of space links. The space links will utilize an "extended IP" including UDP and the Delay/Disruption Tolerant Network standard under development to accommodate distance time delays. The rapid growth in the sizes of on-board memory and storage capabilities of space systems introduces another level of flexibility in scheduling and management of data and data transfers. The space links will utilize the predictability of satellite orbits along with navigation updates to manage antenna coordination and line-of-sight issues. The space link physical and data link layers will use the international CCSDS/AOS standards. The complexity of architecture for a Space SoS that provides communication and navigation services to astronauts and systems on the Moon become apparent in Figure 2 [5]. That figure illustrates several different types of communication links, including: the long-range high data rate trunk lines reaching from the DGSs to the CRSs [6] in lunar orbit (LCRS) and directly to the communication unit on the lunar surface (if that unit is in view of the Earth); the shorter range high data rate links from a LCRS to the surface communication unit or human occupied vehicles; the low data rate links from a LCRS's multiple access subsystems to rovers, robots, science instruments, fuel extraction equipment (in-situ resource unit, ISRU), and astronauts performing extra vehicular activities (EVAs) on the surface; long range surface wireless wide area networks (WANs) that interconnect the surface communication unit, human occupied vehicles, rovers, robots, and network repeaters; and short range surface wireless local area networks (LANs) that interconnect astronauts on EVA, in vehicles, and in habitat, with each other and with rovers, robots and scientific instruments in close proximity. The acronyms DIN, DINCC, DGS, CRS, LCRS and their terms were fabricated solely to describe architecture in this paper 


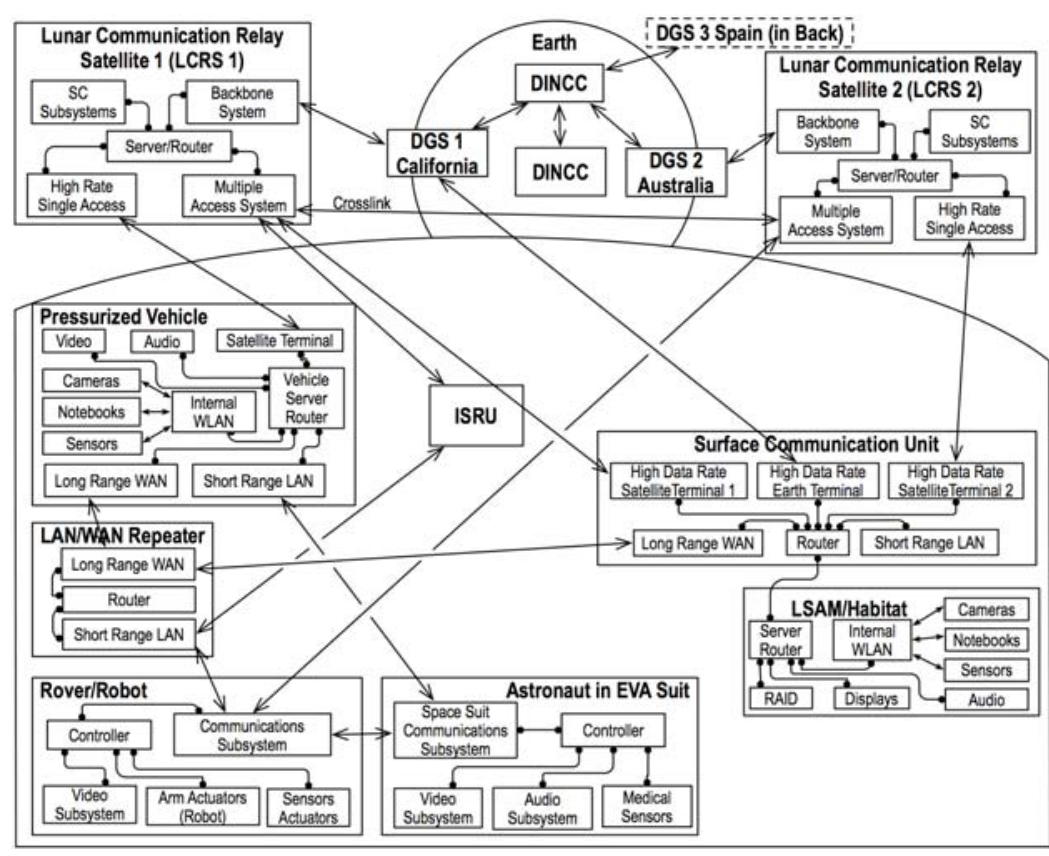

Figure 2. Illustration of the highly complex exploration networks.

the subsystems within each node. The IPcompliant interfaces and routers, prevalent throughout the networks and user nodes, can route data throughout the infrastructure over the tortuous path between any two (or more) nodes on the Moon and/or Earth.

The architecture must describe the joining of these disparate systems so they can intercommunicate and hand off communication links among each other as an exploration mission vehicle passes from one system's domain to another's. Furthermore, the communication services must join so that an exploration customer node may communicate with another node across system domains. The development process for this architecture must lay out the methods, operations, and interfaces needed to join together a federation of multiple, heterogeneous, legacy and new systems that incorporate networks at multiple levels and in multiple domains.

The capabilities of the architecture, driven by emerging exploration requirements, will enable future astronauts to conduct space exploration, communicate with Earth-based

separately from existing legacy architecture and are not terms in general use.

The LANs and WANs enable astronauts to collaborate on their activities by conversing, sharing their data, and sharing their vision by passing video among them while performing construction duties or while exploring. LCRS low data rate links enable communications among astronauts, vehicles, and other surface nodes that are widely spread apart beyond the line of sight of a WAN. The high data rate links enable the Earth operations centers to send voice, video, directives, commands, software, and data to surface nodes on the Moon. Likewise they enable the astronauts and other surface nodes to send voice, video, and science data from exploration activities; health data; navigational location coordinates; and engineering data to interested parties on the Earth.

The complexity of communication on the Moon is further illustrated in Figure 2 by showing the types of networks that may reside within the communication relay and surface nodes. In the figure, the on-board communication subsystems and the control subsystems in a LCRS are interconnected with an IP-compliant network. Each subsystem has its own IP address dispensed by the central computer or server for configuring, operating, and monitoring each of the subsystems on the satellite. The satellites provide IPcompliant communication services on demand of the using nodes and autonomously reduce the need for continuous human operations and scheduling of the services they provide. The other surface nodes in the figure have their own controlling computers/servers and internal IPcompliant networks to interconnect all

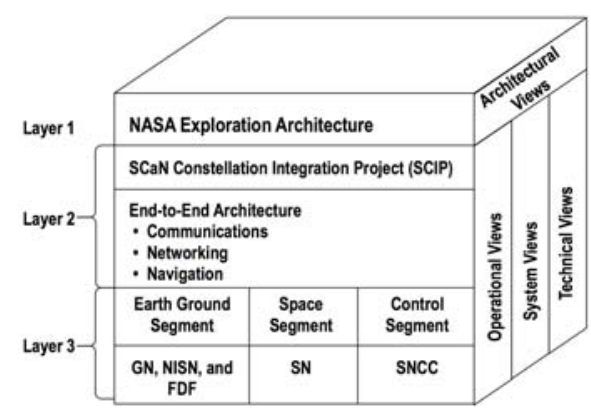

Figure 3, Architecture decomposition scientists, excite future generations of explorers with high definition video to enable a virtual-presence participation in the astronauts' exploration activities, and return safely to Earth at the end of the mission.

\section{Architecture Decomposition Process}

Standard system engineering methods and processes are used for defining and gathering capability requirements, and identifying a generalized initial architecture [7]. Additional steps include decomposing the architecture and using the Department of Defense Architecture Framework (DoDAF) operational, system, and technical view diagrams [8], and then refining the architecture as actual operational activities and functional requirements are identified during the development process [9] [10].

The architecture views convey the space communication and navigation network architecture to all the stakeholders (astronauts, exploration mission customers, system engineers, test engineers, international partners, potential equipment vendors, etc.) to assist them in verifying that the Space SoS will address their concerns or to help them understand the Space SoS and how they may take part in its implementation.

The DoDAF methods of developing the architectural views were chosen for describing the space communication and navigation networks SoS because they are particularly suited to developing and defining complex communication 
architecture and have been vetted by use in defining "as-is" and "to-be" communication architectures for major DoD communication projects. A hierarchical document and diagramming structure is used to present the complex communication and navigation network architecture in an efficient manner to a variety of users with differing needs. Thus, the amount of detail presented increases with each of the successive levels shown in Figure 3. The network architecture is described in terms of its operational, system, and technical attributes so that the user can gain insight into how it fulfills its mission objectives. The network architecture is decomposed into segments and elements. The purpose of the descriptions for each level is to show how segments and elements support the operational, system, and technical aspects of the architecture.

\section{IP-Centric Networks Architecture}

The communication and navigation network architecture must provide end-to-end communications, radiometric tracking, and timing synchronization services at low and high data rates to the crew vehicle and low data rate services to the crew launch vehicle during all mission phases.

The communication and navigation network will consist of the SN, GN, and DSN legacy system elements, the new communication Intranet, and the network elements, previously identified by the fabricated acronyms: DIN, DGS, and the CRS in lunar orbit (LCRS) and later Mars orbit (MCRS), that will be added as space exploration extends into distant and deep space. Some system elements, such as the DSN and the mission control facilities at Johnson Space Center (JSC), have over 25 years of legacy hardware, software, and policies. These networks are administered by different control center software systems and operational policies. To put together an IP compliant end-to-end management system with human rated real-time responsiveness requires stitching together numerous different administrative systems and domains with different policies, priorities, and management systems to form an integrated system that can plan, allocate, control, deploy, coordinate and monitor the resources of the network. Many of the legacy systems supporting existing space missions also have to evolve into the future architecture to meet the space exploration mission requirements. Currently, GN, SN and DSN do not provide a network interface to missions; however, they will need to extend network layer capabilities to space. Quality of Service (QoS) will need to be extended over multiple dissimilar domains. With human lives at stake on Mars or the Moon, automation, automatic error recovery, and local distributed control will have to be pervasive in the architecture.

The capabilities of IP layer-based network architecture are illustrated in Figure 4. The naming of the network layers and the type of data carried over them are shown in the legend at the upper right of the diagram. Starting at the lower left is a scientific instrument that has its own simple internal network that begins at the top of a 5-layer stack, the instrument controlling application layer. This network diagram is concerned with protocol processing, protocol interfaces, and transport mediums. While the protocols reside in system blocks, the internal subsystems are not discussed in detail. This diagram follows the passage of scientific, voice, and video data through protocol layers along a path that provides communication and networking service between the Moon and Earth. In the example, the astronaut or scientist would initiate a voice and video connection with the other. Then the astronaut can initiate the transport of the instrument data by pressing buttons on the instrument or by verbally commanding it to release its data to the scientist's address on Earth. The diagram indicates the paths the instrument data takes through the protocol stacks, routers, and gateways to reach the scientist. These same network paths also handle the voice and video from the astronaut to the scientist in a separate parallel stream. The scientist, in turn converses with the astronaut on the return path.

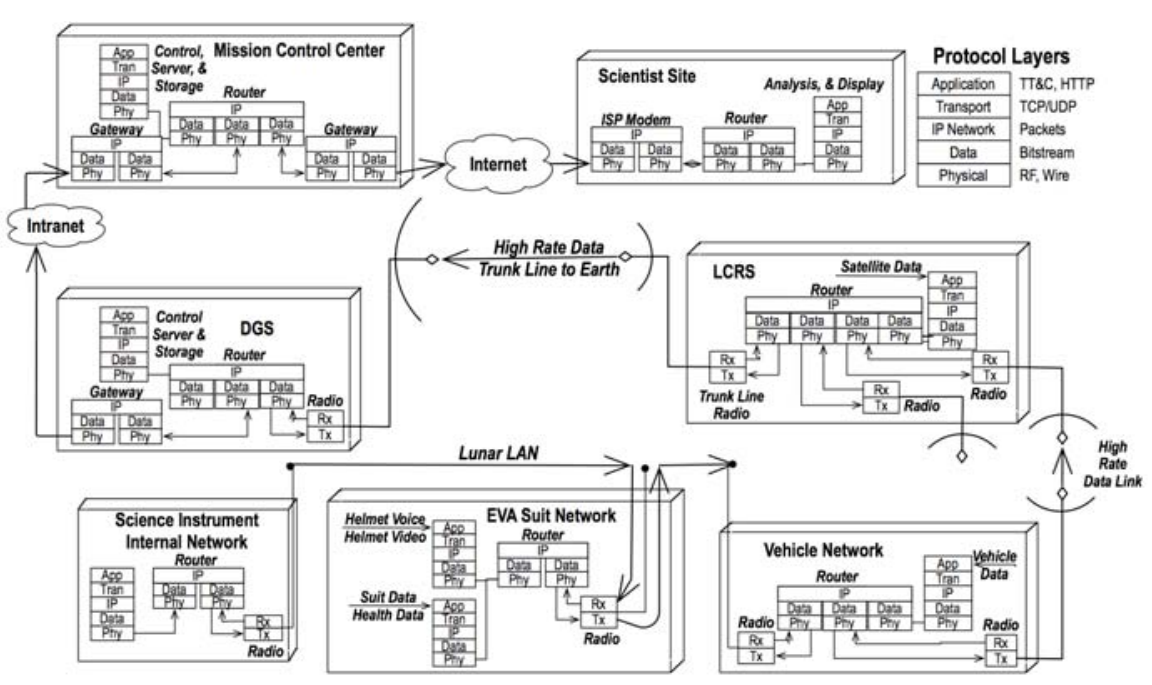

Figure 4, Layered IP Network Connects Ground and Space Together

\section{Propagating Navigation Architecture to Space}

A system of coarse and fine-grain navigation references will be integrated within the Space SoS to enable future space explorers and applications to autonomously access navigation and position services. In other words, astronauts must be able to "fly" crew vehicles and landers independently from an Earth-based mission control center simply because a mission to the Moon or Mars could result in a landing occurring out of sight of Earth. Furthermore, a landing on Mars will occur at a great distance from Earth, so far that real time communication from Earth to Mars is impossible. The astronauts will be in control of their safe landing on Mars, and the networks must provide the communication and navigation services to enable them to act autonomously. 


\section{Applying DoDAF views}

A few typical DoDAF products are described below to illustrate the architecture. Other crosscutting views of the multitude of "as-is" legacy systems, "to-be" future systems, and future IP network decomposition views of the network layers and network-centric architecture may also be useful in describing the architecture. The architecture diagramming methods used range from the defined DoDAF graphical views to custom diagrams that use pictorial icons to represent nodal entities.

The operational view, OV-1, shown in Figure 5, describes the overall operational concept for the architecture being described. Typically, the $\mathrm{OV}-1$ is drawn with icons that represent the various system and operational nodes of that architecture. Here we use a simpler diagramming technique of using labeled boxes to indicate the nodes. The O V - 1 s how s th e operational concept for

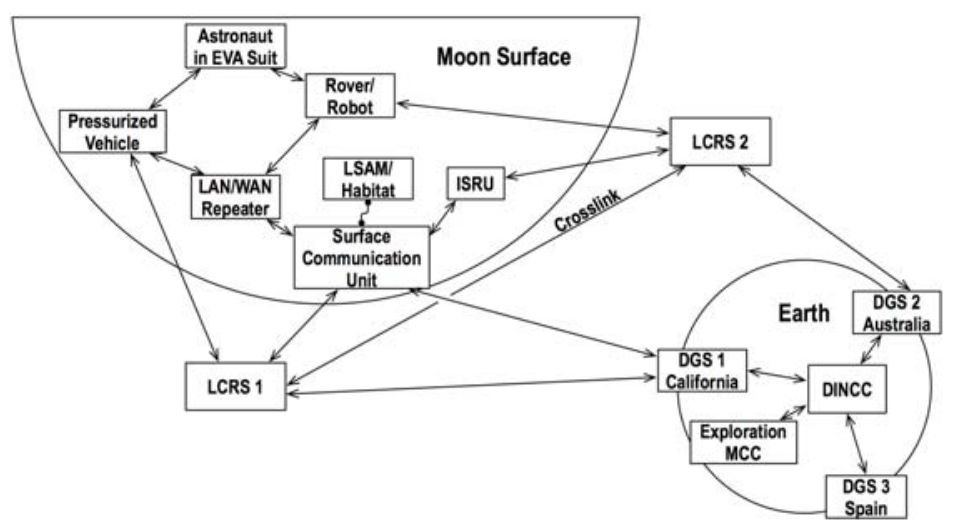

Figure 5. OV-1, Operational concept for lunar exploration represents the customer for the services. The customer interacts with the communication and navigation control center and management by requesting services to support its operations. The communication and navigation control center, in turn, requests network services from, and passes mission customer schedule information to, the DINCC and to the communication Intranet management center. The communication and navigation control center coordinates and schedules the network services with the DINCC that controls the DGS remote sites around the Earth and the LCRSs in orbit around the Moon. The DINCC also monitors the operation and health of the DGS site antennas and the LCRSs. The predictability a n d navigation a 1 verifiability of the LCRSs orbits and the periods of line-of-sight between the LCRSs and the DGS sites enable the possibility of autonomous operation of the Space links. Ideally the operation of the DGS antennas and the LCRSs would be fully autonomous with the DGS antennas maintaining continuous interaction among the DINCC, the DGSs, the Exploration mission control center (MCC), the LCRSs, and the lunar surface nodes. Essentially, the figure indicates that any node may communicate with any other node in its vicinity. What is also implied but not clearly obvious is that the use of IP in the networks enables any node to communicate with any other node on the networks by routing the data through any of the intermediate nodes shown in the figure.

Figure 6 is an OV-4 diagram that shows the relationships among the organizations involved with providing the communications and navigation services to the using nodes. The Exploration MCC shown at the top

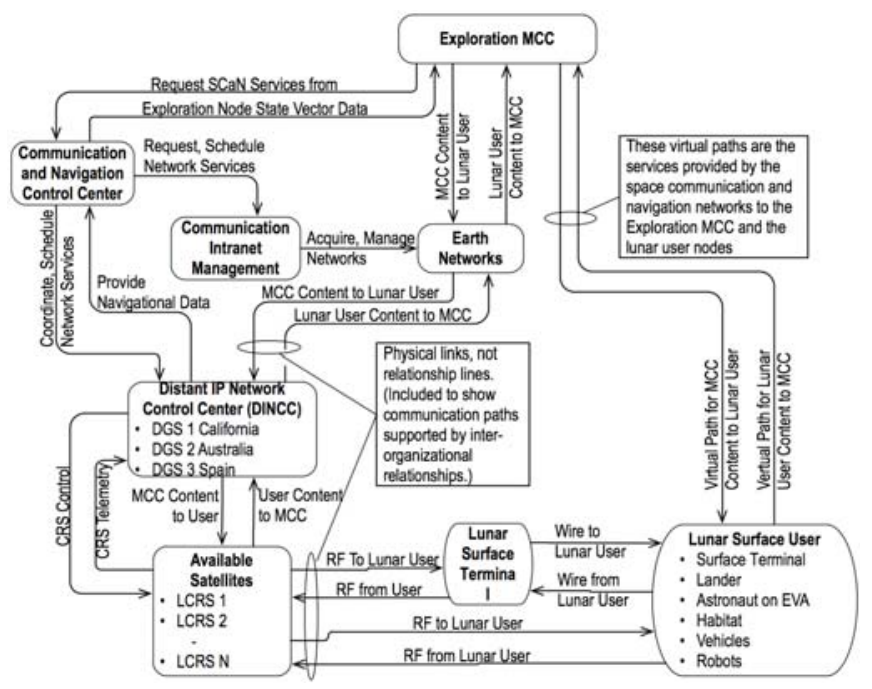

Figure 6. OV-4 Organizational Relationships connectivity with the LCRSs through regular handoffs between stations as they come into and pass out of view of the Moon as the Earth rotates and as the LCRSs may become obscured by the Moon. Likewise, the LCRSs would maintain connection with Earth through the handoffs and would provide lunar surface users with service both scheduled and on demand of the user. The right side indicates the virtual communications paths among the Exploration MCC and the lunar surface nodes provided by the networks. Of course, the real path goes through multiple routing nodes in the network. The bottom of the figure shows that the lunar surface nodes, including the lunar surface terminal, can communicate directly with the LCRSs, for communicating with Earth and/or with other surface nodes. Surface network details are not shown because that network is expected to be fully autonomous and will need no external operations, except for maintenance that can be administered over the network or by astronauts on the surface.

Figure 7 is an OV-2 diagram describing the operational node connectivity for the mission architecture. It is recommended that this diagram be drawn after the organization is defined in OV-4s and after the OV-5 activities (not shown) are identified. Then the information that each organization needs to do its work can be identified along with the source of the information. The rectangular boxes in the diagram indicate the operational nodes. Inter-nodal connections are called needlines and the rounded rectangles display the information that must be passed from one node to another to enable each node to accomplish its operational tasks. For example, an astronaut who is communicating with a scientist on Earth may wish 


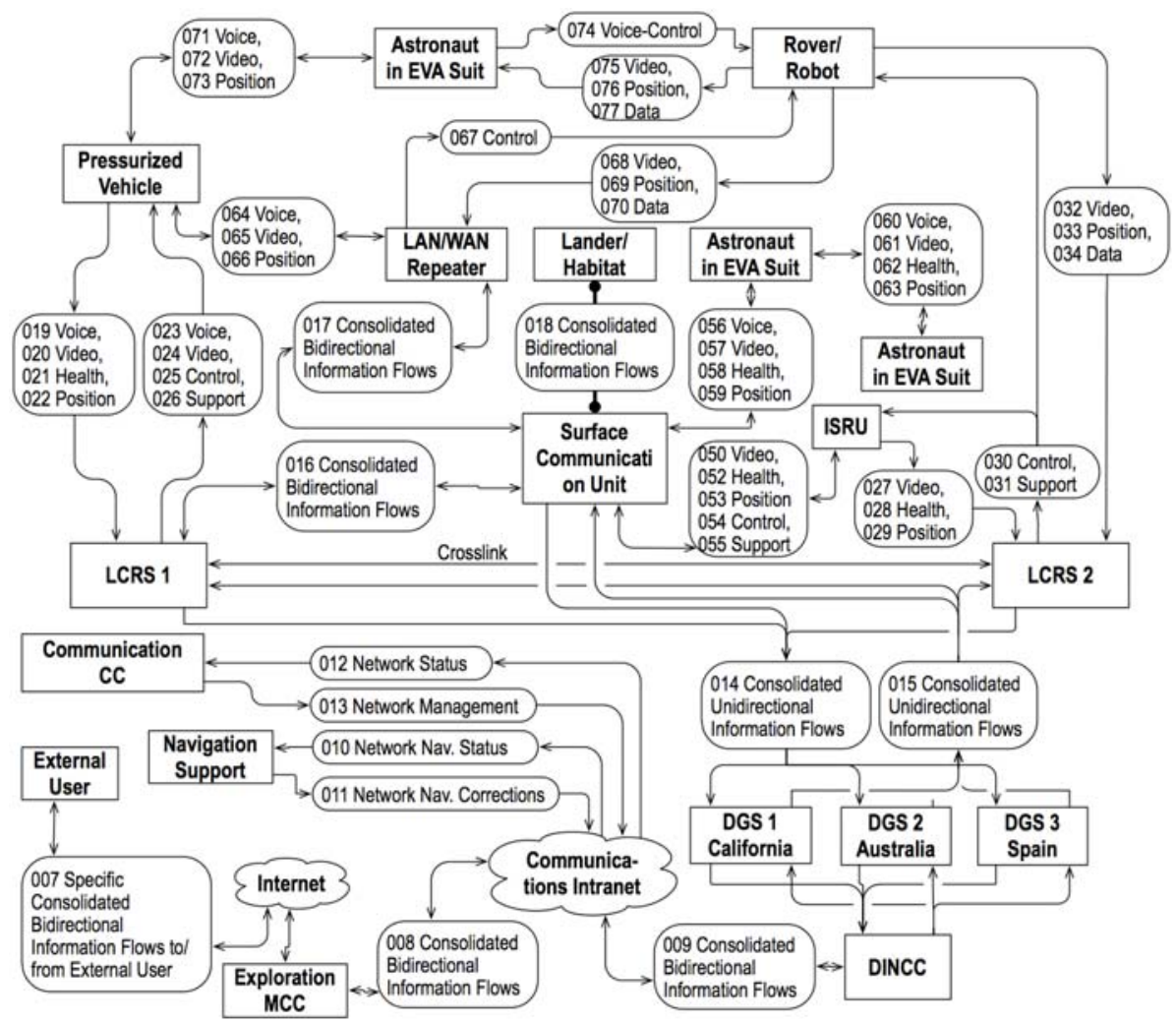

Figure 7. OV-2, Operational node connectivity diagram
DIN system. Likewise, the LCRSs are aggregated as components of a larger LCRS Constellation. It's possible to make this simplification if all the satellites in the constellation are identical. If not, they should be treated as unique individuals in the diagrams. The purpose of SV-1 is to identify all the interfaces among all the system nodes; here they are numbered. SV-1 leads into SV-2 (not shown), which is a similar diagram that shows the type of communication that occurs on each interface line, such as radio frequency band or network type. Ultimately, a system data exchange matrix (SV-6) is constructed that contains all the pertinent information that can be identified or decided during architecture development that passes over each interface. Included for each interface in the matrix is the needline and operational node information (obtained from the OV-2) that passes over the interface. It is from this matrix that the system engineers can extract the requirements for the interfaces. to show the scientist interesting mineral deposits. Such a communication path might be as shown in the figure where the astronaut communicates with voice, microscope video of the minerals, and data readout of a portable analysis device, such as an $\mathrm{x}-$ ray fluorescence instrument that is passed from the astronaut's helmet radio to the vehicle shown. The vehicle aggregates of all the data in its vicinity and passes that to an overhead LCRS. The LCRS forms a larger aggregate of all the data passed to it and sends the data that is addressed to Earth on to an Earth ground station. The ground station extracts the IP data and sends it on to the Exploration MCC that, in turn, routes the voice, video, and data on to the scientist, who may be accessing her end of the connection over the Internet. Communication from the scientist is returned on the same path. Many possible communication paths among any and all nodes can be constructed from figures similar to the one shown.

Figure 8 shows an example of the system interface diagram (SV-1) for the communication and navigation systems and services needed to support the activities of nodes on the surface of the Moon. This diagram uses UML component (rectangle with a small component artifact in the upper right corner) and system (block-shaped) icons to describe the Space SoS layout. The little rectangles indicate ports into and out of a system block. In the diagram, the ground stations and DINCC are shown as components of the larger

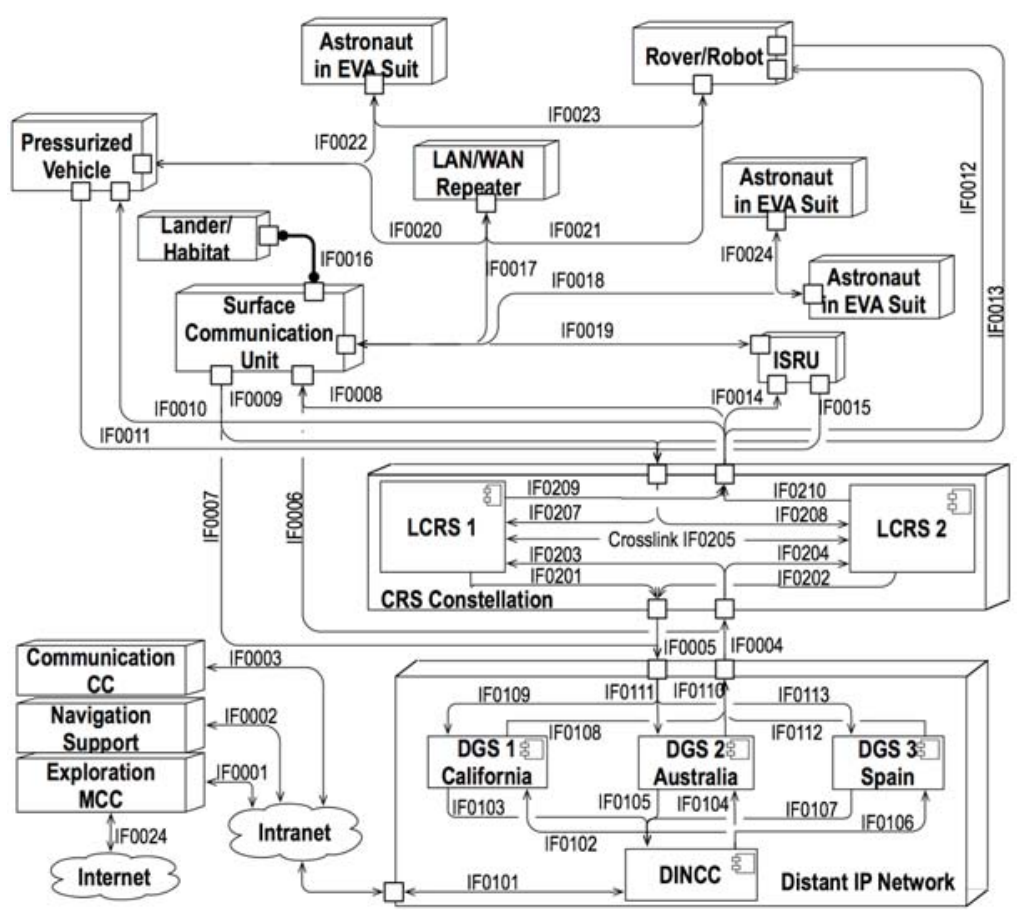

Figure 8. SV-1 Systems and services interface description 


\section{Modeling, simulation and system engineering}

Extensive modeling and simulation is necessary for development and test of the interoperation among the networks and systems that is needed to save cost and increase reliability.

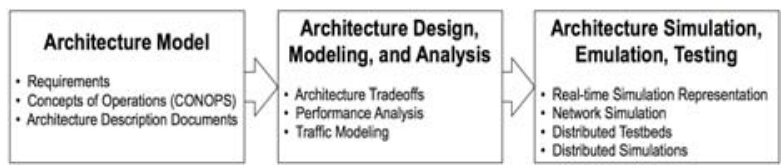

Figure 9, Architecture modeling and testing process.

A top-level view of the architecture-modelingsimulation process for the communications and navigation networks Space SoS is shown in Figure 9. The use of an architecture framework for the communications and navigation networks allows the creation of the architecture definition documents that contains diagrams and tabular data sets needed as input for the architecture modeling and analysis. Data traffic models and analysis is needed to optimize the data flow among the SoS, especially for the legacy space communications and navigation networks. The network simulation and emulation tools based on space and terrestrial protocols are being developed and used. Distributed simulation testbeds are being developed for the Space SoS during the formulation design phases.

\section{Conclusion}

Communication and navigation networks are essential elements of Space SoS. Their level of involvement can range from a few space links to a complex infrastructure or SoS. The challenges presented by the architecture of the communication and navigation networks are:

- Implement high reliability, utilizing standard protocols;

- Utilize the advantages of IP to provide transparent connectivity and interoperability among nodes on the networks;

- Encapsulate IP over Space links maintaining International Compatibility;

- Incorporate proven Space link technology (Upgrades Legacy Network Technology);

- Implement autonomous network management to reduce the cost of operations;

- Utilization of Architecture Frameworks to provide operational, system, and network interface views of a complex Space SoS interconnected with a Network of Networks.

\section{Acknowledgements}

The authors would like to thank Charles Putt and Lee Jackson of the Glenn Research Center for their thorough reviews and edits of the paper.

\section{References}

[1] J.L. Hayden, "Spacecraft/ground Architectures Using Internet Protocols to Facilitate Autonomous Mission Operations. Proceedings of the 2000 IEEE Aerospace Conference," March 2000. Available from the IEEE $0-7803-5846-5 / 00$

[2] K.B. Bhasin and J.L. Hayden, "Inter-Spacecraft Communication Architectures and Technologies for Coordinated Spacecraft Missions," AIAA 2001 Space Conference and Exposition, Albuquerque, NM, 28-39 August 2001. Available from the AIAA 2001-4709

[3] K.B. Bhasin, T. Linsky, J.L. Hayden, and S. Tseng, "Surface Communication Network Architectures for Exploration Missions," AIAA Space 2005, Long Beach. Available from the AIAA-2005-6695

[4] K.B. Bhasin and J.L. Hayden, "Space Internet Architectures and Technologies for NASA Enterprises.” Int. J. Satell. Commun. 2002; 20: 000000 (DOI: 10.1002/sat.727)

[5] K.B. Bhasin, A.W. Hackenberg, R. Slywczak, P. Bose, M. Bergamo, and J.L. Hayden, "Lunar Relay Satellite Network for Space Exploration: Architecture, Technologies and Challenges," Proceedings 24nd AIAA International Communications Satellite Systems Conference, 12-14 June, 2006, San Diego, CA. Available from the AIAA-2006-5363

[6] J. Soloff, D. Israel, and L. Deutsch, “A Sustained Proximity Network for Multi-Mission Lunar Exploration," AIAA 1st Space Exploration Conference, Orlando, FL, Jan 30-Feb 12005. Available from the AIAA-2005-2505

[7] M. Maier, "Architecting Principles for Systems-ofSystems," System Engineering, Vol. 1, No. 4, pp 267-284, 1998.

[8] K. Bhasin, C. Putt, J. Hayden, S. Tseng, A. Biswas, B. Kennedy, E. Jennings, R. Miller, J. Hudiburg, D. Miller, A. Jeffries, and T. Sartwell, "Architecting the Communication and Navigation Networks for NASA's Space Exploration Systems," 2007 IEEE International Conference on System of Systems Engineering, San Antonio, TX, April 16-18, 2007. Available from the 2007 IEEE 1-4244-1160-2/07

[9] DoDAF V. 1.0, 2003, DoD Architecture Framework Version 1.0, the Deskbook and Volumes I \& II, Department of Defense Architecture Framework Working Group. Superceded by DoDAF V. 1.5.

[10] DoDAF V. 1.5, 2007, DoD Architecture Framework Version 1.5, Volume I, II and III, Department of Defense Architecture Framework Working Group. Available at https://dars1.army.mil/IER/index.jsp, Accessed on 1 Dec 2007. 